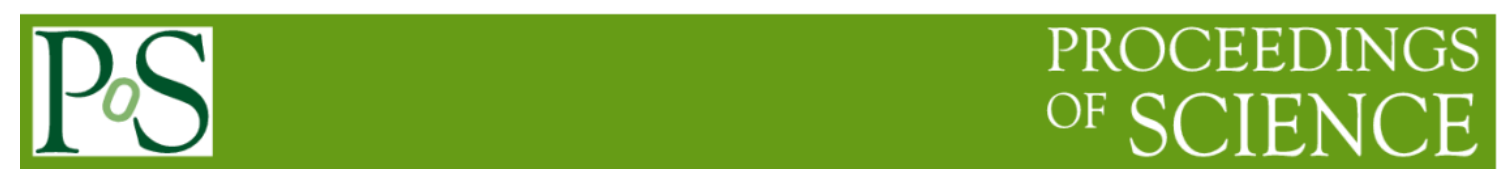

\title{
Wrap-up and thanksgiving
}

\section{Zdeněk Doležal *}

Charles University, Faculty of Mathematics and Physics

V Holešovičkách 2, Prague, The Czech Republic

E-mail: doleza@ipnp.mff.cuni.cz

This is the closing talk of the conference given by the International Advisory Committee chair.

40th International Conference on High Energy physics - ICHEP2020

July 28 - August 6, 2020

Prague, Czech Republic (virtual meeting)

*Speaker

(C) Copyright owned by the author(s) under the terms of the Creative Commons 
Ladies and gentlemen, dear colleagues, dear guests,

This is the closing talk of the 40th International Conference on High Energy Physics. It has been very different from a normal conference but let me hope that it was interesting and useful. And as usual: it could not happen without strong support and tireless effort of many people and institutions. It is impossible to list them all, but I tried (see Figures 1 and 2 and Table 1) and already now apologise to all not listed.

At the end I have to thank the $\mathrm{C} 11$ commission: we have been given a second chance: ICHEP2024 has been assigned to Prague and let's hope it will be a standard one.

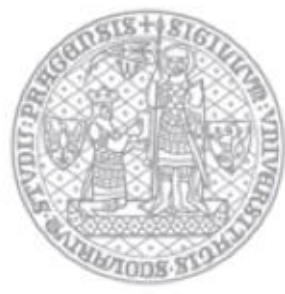

Charles

University

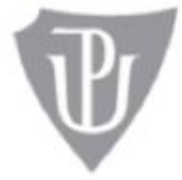

Palacky University

Olomouc

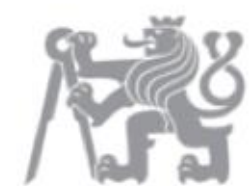

Czech Technical University in Prague

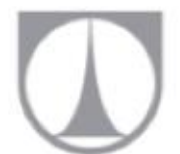

Technical University

Liberec

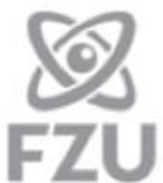

Institute of Physics Croch Acodemy of Science

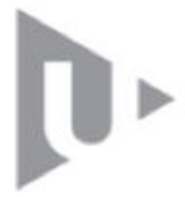

University of Western Bohemia Pilsen

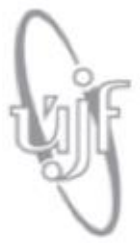

Nuclear Physics Institute Crech Academy of Sciences

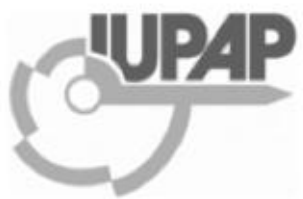

International Union of Pure and Applied Physics

Fig. 1: Organising institutions 


\begin{tabular}{|c|c|c|c|c|c|c|}
\hline Halina Abramowicz & Jiñ Chudoba & Takanori Hara & Vladimir Kokolia & Marcela Mikesstiková & Femando Quevedo & Tomás Syykora \\
\hline Dagmar Adamová & Petr Chudoba & Despina Hatzifotiadou & Manàn Kolesár & Joachim Mnich & Jîi Rameš & Petr Tas \\
\hline Markus Ahlers & Jiñ Chýla & Jiri Hejbal & Petra Köppl & Jocelyn Monroe & Pavel Reznicek & Marek Taševskì \\
\hline Federico Antinori & Giacomo Cuttone & Stuart Henderson & Mchal Koval & Clemencia Mora Herrera & Jan Ridkỳ & Geoffrey Taylor \\
\hline Aram Apyan & Anne Evelyn Dabrowski & JoAnne Hewett & Robert Kralik & Bemdt Mue ller & Lenny Rivkin & Mark Thomson \\
\hline Kamil Augsten & Valerio Dao & Jan Hladký & Filip Kñżek & Marcelo Munhoz & Natalie Roe & Tomas Tmej \\
\hline Jonathan Bagger & Tomás Davidek & Michael Hoch & Jiti Kroll & Cartos Munoz Camacho & Andrea Rossi & Sarka Todorova \\
\hline Elisabetta Barberio & Amol Dighe & Yonit Hochberg & Susanne Kuehn & Hitoshi Murayama & Martin Rybar & Mia Tosi \\
\hline Petr Baron & Caterina Doglioni-Fitzpatrick & Jini Hofejsi & Alexander Kupčo & Miroslav Myska & Heidi Schellman & Petr Trávnićek \\
\hline Matt Barrett & Jiñ Dolejsi & Jing Huang & Alexander Kuzmin & zástupce MZV & Martin Schnabl & Jaroslav Tmka \\
\hline Ursula Bassler & Zdenếk Doležal & Zdenék Hubacek & Jiñ Kvita & Marlene Nahrgang & Thomas Schwetz & Barbara Trzeciak \\
\hline Ignácio Bediaga & Laura Fabbietti & Matej Hudec & Marzena Lapka & Tatsuya Nakada & Silvia Scorza & Nikolai Tyurin \\
\hline Prafulla Behera & Gabriel Facini & Tomáš Husek & Yen-Jie Lee & Yu Nakahama & Ettore Segreto & Patricia Vahle \\
\hline Ivan Belyaev & Jana Faltovà & Atsuko Ichikawa & Rupert Leitner & Mihoko Nojiri & Hiroyuki Sekiya & Ruth van de Water \\
\hline Michael Benedikt & Torben Ferber & Toru lijima & Jianglai Liu & Tomas Nosek & Liz Sexton Sexton Kennedy & Luboš Veverka \\
\hline Stan Bentvelsen & Afturo Femandez Tellez & Artem Isakov & Nigel Lockyer & Jiri Novotny & Constaninos Skordis & Antonio Vilela Pereira \\
\hline Stefania Beolé & Lukás Fiala & Akimasa Ishikawa & Kristin Lohwasser & Yukiyoshi Ohnishi & Karel Smolek & Vit Vorobel \\
\hline Jaroslav BielĊik & Renato Fonseca & Paul Jackson & Roman Lysák & Yasuyuki Okumura & Walter Snoeys & Václav Víba \\
\hline Jana Bieléíkovả & Juan Fuster & Kart Jakobs & Julie Malcles & Arantza Oyanguren & Stefania Sôldner-Rembold & Yifang Wang \\
\hline Agnese Bissi & Jie Gao & Katerina Jarkovska & Mchal Malinský & Josef Pácalt & Alex Sousa & David Waters \\
\hline Jiri Blazek & Hedvika Gedeonová & Tomas Jezo & Michal Marcíšsovský & Pablo Pais & Karel Soustružnik & Risa Wechsler \\
\hline Martina Bohácová & Vjačeslav Georgiev & Jan Kadełábek & Daniele Margaronne & Inkyu Park & Michel Spiro & Elizabeth Worcester \\
\hline Radja Boughezal & Yvonne Geyer & Karol Kampf & Ioana Maris & Giovanni Passaleva & Martin Spousta & Qun Wu \\
\hline Concezio Bozzi & Paolo Giacomelif & Jakub Kandra & Maria Alday Martin & Mitesh Patel & Martin Spousta & Masanori Yamauchi \\
\hline Pierluigi Campana & Fabiola Gianotti & Iurii Karpenko & Karim Massi & Ryan Patterson & Sally Ŝtefková & Alexey Yushkov \\
\hline Florencia Cannelli & Gian Giudice & Pavel Kasik & Victor Matveev & Elisabetta Pennacchio & Graeme Stewart & Jaroslav Záleșák \\
\hline Ina Carí & Carla Göbel & Jihn E. Kim & Patricia McBride & Dennis Perepeiltsa & Miroslav Sulc & Michal Zamkovsky \\
\hline Jakub Ceska & Steven Goldfarb & Ingrid Knapova & Petra Merkel & Voftéch Petrácek & Michal Sumbera & Ota Zaplatilek \\
\hline Lukás Chlad & Sowjanya Gollapinni & Anders Knospe & Lia Merminga & Tadeás Petrú & Michal Svatos & Iveta Zatoćlová \\
\hline \multirow[t]{2}{*}{ Philippe Chomaz } & Stefania Gori & Peter Kodys & Daniel Mihatsch & Souvik Priyam & Martin Sýkora & Alfonso Zerwekh Arroyo \\
\hline & & & & & & Radek Zlebcik \\
\hline
\end{tabular}

Fig. 2: List of people who contributed significantly to the organization

\begin{tabular}{|l|l|}
\hline BNL & Jablotron \\
\hline CERN & JINR \\
\hline DESY & KEK \\
\hline GSI Darmstadt & MSMT \\
\hline IHEP Beijing & $\begin{array}{l}\text { Centre of Advanced Applied Sciences } \\
\text { (co-financed by EU) } \\
\end{array}$ \\
CZ.02.1.01/0.0/0.0/16 019/0000778 \\
\hline IN2P3 & \\
\hline
\end{tabular}

Tab. 1: Contribution of these sponsors is acknowledged. 